UF JUR 2018/2019 Editorial Board:

* also served as Associate Editors

Rocio Amorin

Arora Harneet

Martha Battaglin Ramos

Krishna Bhattarai

Deanna Bousalis

Julie Bray*

Mark Chatfield

Rodquel Chism

David Christianson

Peter Chutcharavan*

\section{Matt Conlin*}

Joshua Davis

Raj Desai

Andrea Fidler

Nicholas Genna

Shabnam Goli

Tayler Hansen

Rachel Hartnett

Elizabeth Hartsell

Bryan James*

Amanda Kastner
Shea Keene

Larios Mendieta

Isabel Laurenceau

Peter Licari

Julia Maki

Devon Mims*

Florencia Otegui*

Ayodeji Otufowora

Venkata Pantangi

Emily Pappo

Caroline Reed

Patrick Rizk

Debalina Saha

Tia Tyler

Valerie Valerio-Holguín*

Krista Vaught*

Jamie Williams 